tions that it slows down very considerably after weaning.

It may be mentioned that external parasites which are rare on Blue and Fin whales at South Georgia, are abundant on those taken at the Cape, Pennella being particularly plentiful. Many of the whales examined at Saldanha Bay were also notable for the numerous and peculiar scars which are found on the body, mostly towards the tail. These are in the form of clean, cup-shaped holes in the blubber, and in the majority of whales they are found in all stages of healing. Old scars are quite common in this position on whales at South Georgia, but the unhealed pits are very rare.

\title{
The Problem of Artificial Production of Diamonds.
}

\section{$\mathrm{T}$} E relations between the allotropic forms of carbon constitute one of the most baffling problems in chemistry. The hardness and incompressibility of the diamond point to its structure as being an exceedingly stable one, and the examination by $\mathrm{X}$-rays proves it to have the tetrahedral lattice which accords with the known chemical properties of the carbon atom. On the other hand, graphite has its atoms arranged in sheets made up of hexagons, the carbon atoms in that plane being actually closer than in the diamond ( 1.42 instead of 1.53 Angstrom units), but the sheets are widely separated, their distance being $3.41 \mathrm{~A}$., so that the structure is a loose one, corresponding with the easy cleavage. Graphite is readily produced from diamond at high temperatures, but the converse change has presented the greatest difficulties, graphite having shown itself under all conditions to be the more stable modification. It has been supposed that the atoms of carbon in the two substances differ in theirelectronic structure, the atom in diamond having four $2_{1}$ orbits, and that in graphite only three $2_{1}$ orbits and one of the $2_{2}$ form, an arrangement which is consistent with the two space lattices and also with the great difference in electrical conductivity between the two modifications. It is doubtful whether specimens of graphite have ever been obtained quite free from other elements, and Prof. H. E. Armstrong has maintained that it always contains hydrogen, which is the cause of its open structure.

The diamond is found in Nature under conditions which afford little clue as to its genesis, but as it is converted into graphite by heat in the laboratory, it has seemed probable that it has been formed under a high pressure, and most attempts at its artificial production have started from this assump. tion. Of these, the most famous were those of Prof. Henri Moissan. Moissan's most successful experiments were made by melting pure iron with sugar charcoal in an arc furnace, and plunging the crucible containing the molten mass into cold water. The pressure produced by the rapid chilling of the outer crust was relied on to bring about the conversion of the carbon, so that it would crystallise from its solution in iron as diamond, and not, as usual, as graphite. The iron was successively treated with nitric and hydrochloric acids, with oxidising mixtures, and with fused potassium hydrogen fluoride to remove all minerals except diamond, and after the operation minute crystals were found, some of which had the optical proper- ties of diamond and yielded carbon dioxide on combustion. Molten silver was also used as a solvent, with similar results. Sir William Crookes, working with the residues found in a bomb in which cordite had been exploded, the pressure being calculated to reach 8000 atmospheres, obtained similar crystals after the same chemical treatment. Crookes arrived at the conclusion that carbon, if sufficiently heated under a pressure of 2350 atmospheres, would be liquefied, behaving in this respect like arsenic, and should then crystallise on cooling.

Sir Charles Parsons carried out extensive experiments on this subject, the results of which were communicated in his Bakerian Lecture of the Royal Society for 1918. The main conclusions were :

1. That carbon, if melted or vapourised under a pressure of 15,000 atmospheres does not crystallise, as predicted by Moissan and Crookes.

2. That Moissan's supposition that great pressure was produced by quenching molten cast iron is erroneous.

3. That if the crucible, instead of being quenched, were subjected to a pressure of 100 tons per in. ${ }^{2}$ in a steel die under a press, less crystal residue was obtained.

4. That impurities in the iron, such as silicon, aluminium, magnesium, and chromium, greatly increase the amount of crystalline residue, whilst commercially pure iron melted and carburised with graphite yielded practically no residue.

All attempts to produce diamond by the rapid compression and adiabatic heating of acetylene and other substances (as by firing rifle bullets into cavities which they fitted closely), without the use of iron as a solvent, failed to give a crystalline residue.

The method of analysis adopted was that used by Prof. Moissan. Great uncertainties are involved, as there are no chemical reagents which can be relied on to dissolve every crystalline substance formed at such high temperatures other than diamond. Some of the minerals are extremely resistant to acids, even after prolonged boiling, whilst fusion with alkalies cannot be used, as it destroys diamond. The only certain test is that of combustion, microscopical examination heing uncertain in view of the hardness and transparency of some of the spinels and carbides. When the crystals are very minute, all that can be done is to place them in a silica boat and heat in a current 
of oxygen, watching for a luminous flash when combustion occurs.

According to Prof. Le Chatelier, Moissan's supposed discovery has not been generally accepted in France, but at the time of the Bakerian Lecture his authority was such that Sir Charles Parsons accepted the statement that diamonds had been obtained, although he found it necessary to correct a number of details in the original account. Crookes also, speaking with high authority, confirmed the statements of Moissan, and described crystals which he regarded as being undoubtedly diamonds. $\mathrm{He}$ found one crystal, in particular, to show octahedral planes with dark boundaries due to a high index of refraction. He was able to distinguish such crystals with ease from those of carborundum, which are doubly refracting, but optical appearance alone would not serve to distinguish them from minerals of the spinel group. Unfortunately, although Moissan states in his book on the electric furnace that he prepared many artificial diamonds, no microscopical slides containing them are known to exist. The sketches reproduced by Crookes in his little book on diamonds only indicate that the crystals are of octahedral form and of high refractive index.

In view of the doubts which have been expressed as to Moissan's results, Mr. H. M. Duncan, who was associated with Sir Charles Parsons in his experiments, has repeated very carefully the various analytical processes, and his observations have been placed at our disposal by Sir Charles Parsons. It is found that spinels formed at a high temperature from magnesia and alumina, for example, are exceedingly resistant to repeated treatment with concentrated hydrofluoric and boiling sulphuric acids, and being colourless and crystallising in the regular system, they may readily be mistaken for diamonds. When a residue containing crystals of this kind is placed in a platinum boat and heated in a stream of oxygen in a tube of transparent silica, flashes are often observed, but the crystals of spinel are found to be unchanged. The flashes are to be attributed to carbon dust, which is often present in the air to such an extent that when an apparently clean boat is placed in the tube, many flashes are still seen.

Whenever Moissan's experiments with cast iron have been repeated, although many particles resembling diamond in appearance have been obtained, they have never withstood the combustion test. In order to prove that the methods adopted for removing foreign substances are not too severe, five very small fragments of boart have been placed in a platinum dish, and their size and appearance under the microscope noted, after which they were mixed with $15 \mathrm{gm}$. of iron filings and about a gram of graphite. The mixture was then subjected to chemical treatment until the whole of the iron and graphite had been removed. Microscopical examination showed that the particles of boart had not been destroyed, so that had similar particles been present in any of the residues from cast iron fusions they would not have been lost.
When repeating the experiments, the most characteristic residues were selected for combustion, and a blue pencil used to mark the position of each small crystal in the silica boat under the microscope, before placing it in the combustion tube. After heating at $900^{\circ}$ in oxygen, which had been previously filtered through cotton wool, the boat could be again placed under the microscope. In one of these tests, five very characteristic crystals, photographs of which had been exhibited by Sir Charles Parsons at the Royal Society soirée in 1918, were tested in this way, and were found to be unburnt.

It is possible that Moissan's residues had become contaminated during the long period of their preparation. It is stated that in order to obtain sufficient material for a quantitative combustion test, no less than 80 ingots were made and dissolved. As the extraction with successive reagents is a most tedious process, there was ample opportunity for accidental contamination with carbon in the course of the operations.

Sir Charles Parsons and Mr. Duncan have also repeated the experiments of other workers who have claimed to have produced diamonds by artificial processes, but in every case the results have been negative. The conclusion seems inevitable that diamonds have not yet been produced in the laboratory, and that investigators have been misled into regarding as diamonds various transparent, singly - refracting minerals which happen to be very resistant to chemical reagents.

When graphite is heated to very high temperatures under pressures as great as 15,000 atmospheres, it remains perfectly soft, and there is no indication of the production of a new phase. This was confirmed by the experiments of Sir Richard Threlfall, published in 1908. The pressure produced in quenching molten cast iron in water or lead is not remarkably high, being limited by the yielding of the iron shell under stress, and the supporters of Moissan's views have therefore supposed that the action was not one of pressure, but of crystallisation from a solvent, which might be expected to give the less stable phase, whilst prolonged heating would convert it into the more stable graphite. Success had been claimed by Friedlander for experiments in which fused olivine was used as the solvent; but a repetition of the fusions, described in the Bakerian Lecture, gave negative results, and with such a solvent the chance of producing crystals which might be mistaken for diamond on a casual inspection is evidently great. Inconclusive experiments were made by 0 . Ruff in 1917, which showed that minute diamonds introduced into heated hydrocarbons under high pressure did not increase in size, but minute crystals which might have been diamonds were obtained from carbon in metallic solvents. The sources of error in all such observations having been detected, it must be admitted that the origin of the diamond in Nature remains an unsolved problem.

C. H. D. 